\title{
A MICROMACHINED TORSIONAL MIRROR FOR TRACK FOLLOWING IN MAGNETO-OPTICAL DISK DRIVES
}

\author{
Joe Drake and Hal Jerman \\ Seagate Research \\ 1870 Lundy Avenue, San Jose, CA 95131-1826
}

\begin{abstract}
An electrostatically actuated polysilicon surface micromachined torsional mirror has been used as a fine actuator for track following in Optically Assisted Winchester (OAW'M) magneto-optical disk drives. OAWTM technology combines a Winchester-style voice coil motor (VCM) as a coarse actuator with light delivery via optical fibers and a read/write head containing microfabricated optical elements, a coil, and a silicon chip containing the micromachined mirror. The mirror achieves a resonance of at least $35 \mathrm{kHz}$, an angular deflection range of $+/-2$ degrees prior to electrostatic pull-in, and a pull-in voltage less than 150 Volts. Novel fabrication techniques allow for an RMS roughness of less than $3 \mathrm{~nm}$ on the reflecting surface of the mirror and mirror plate radius of curvature of greater than $10 \mathrm{~cm}$ over a temperature range of 5 to $55 \mathrm{C}$. Servo bandwidth for OAW disk drives has been demonstrated at $3-5 \mathrm{kHz}$ while using the mirror for track following at greater than 35 thousand tracks per inch (ktpi).
\end{abstract}

\section{INTRODUCTION}

In the continuing effort to increase data storage density, the use of a dual stage servo system in hard disk drives has received considerable research attention. Several researchers $[1,2]$ have described the use of a piezoelectric push-pull actuator mounted between the coarse actuator arm and the head suspension to finely position the entire suspension and head assembly over the data tracks. Typically the reported servo bandwidth of such arrangements is on the order of a few $\mathrm{kHz}$. Imamura, et. al. [3] demonstrated a micromachined suspension/slider concept wherein the entire slider is translated in the cross-track direction by a parallel plate electrostatic actuator. The reported bandwidth of this actuator was $34 \mathrm{kHz}$ (without the slider present) with an actuation voltage of 50 Volts and a maximum displacement of $0.55 \mu \mathrm{m}$ but the system servo bandwidth was not reported. I Iorsley, et. al. [4] also used a parallel plate electrostatic micromachined actuator for fine translation of the slider $( \pm 1 \mu \mathrm{m})$ at \pm 40 Volts. This actuator was constructed of polysilicon and the resulting servo bandwidth of the dual-stage system was $1.2-2.4 \mathrm{kHz}$.

Magneto-optical (MO) data storage presents the possibility of an elegant solution to the need for a second stage actuator. For MO data storage the fine actuator need only steer the focal point of the optical spot rendering unnecessary the movement of relatively massive components such as suspensions or sliders. Sekimura, et. al. [5] recognized this feature and described a bulk micromachined torsional mirror as a fine actuator mounted on an optical head in an MO data storage system. This mirror had an angular rotation range of $\pm 0.5 \mathrm{mrad}$ ( \pm 0.03 degrees) for an actuation voltage of 5 Volts and a bandwidth in excess of $50 \mathrm{kHz}$.

Optically Assisted Winchester (OAWTM) [6] technology presents a data storage approach whereby the familiar and welldeveloped advantages of conventional Winchester style disk drives are integrated with optical elements that provide for MO data storage. In OAW ${ }^{\mathrm{TM}}$ technology, a rotary voice coil motor (VCM) actuator is used for coarse positioning of a suspension-mounted $\mathrm{read} / \mathrm{write}$ head. Laser light is delivered to the head via an optical fiber mounted onto it (see Figure 1). A micromachined torsional mirror is mounted onto the trailing edge of the head and reflects the laser light that emanates from the fiber thus redirecting it normal to the MO storage media on the disk surface. Before reaching the disk, the laser spot is focussed through an aperture in a magnetic coil by a microfabricated-molded lens. By rotating about the axis defined by the torsional hinges, the reflective mirror plate translates the position of the focussed laser spot in the cross track direction thus providing the fine actuation for the dual-stage servo system (see Figure 2). Data is written to the MO media by heating the media to the Curie point with the laser spot while the coil-generated magnetic field is applied. Data is read back using the Kerr effect; laser light reflected off of a written spot in the media undergoes a slight rotation in its polarization compared to the reflection off of an unwritten spot. Thus the polarization of the reflected light determines the bit polarity.

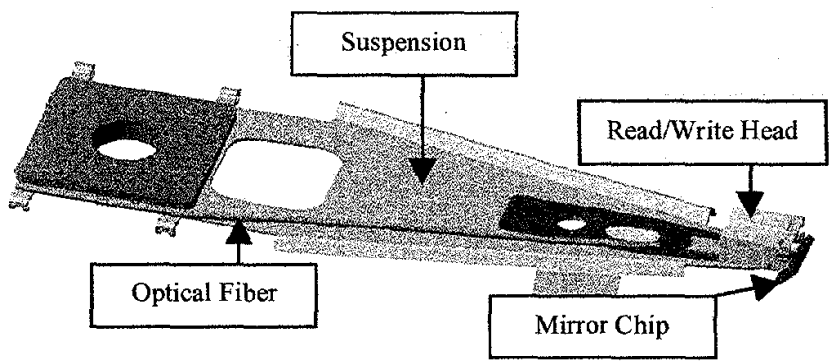

Figure 1. OAWTM Suspension assembly with read/write head and optical fiber. Micromachined mirror is fabricated onto the mirror chip (drawing courtesy of Mike Darling).

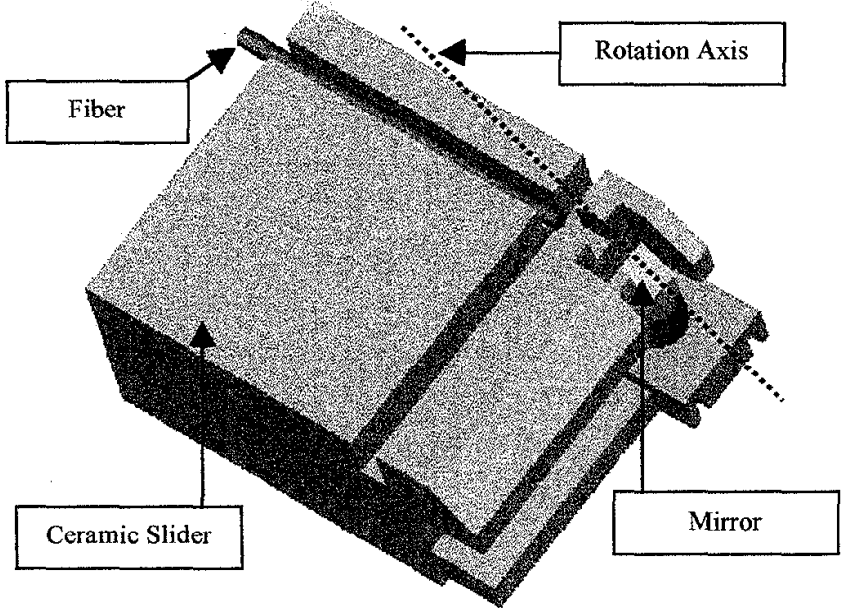

Figure 2. Schematic diagram showing how the torsional mirror rotation steers the optical spot. The supporting chip is stripped away in this view revealing the location of the mirror plate.

The use of the Winchester hard drive architecture allows for the presence of many such read/write heads in a single disk drive as well as the ability to store data on both sides of each disk. An optical switch [7] is used to select the active head by switching the beam from a single laser source to the optical fiber routed to the appropriate head. 


\section{TORSIONAL MIRROR DESIGN}

\section{Optical Properties}

For optical efficiency, the reflecting surface must be smooth, flat, and highly reflective at the laser wavelength of $650 \mathrm{~nm}$. To achieve optical smoothness, the polysilicon mirror plate is polished as described in the following fabrication sequence. A $100 \mathrm{~nm}$ thick Au layer affixed to the polysilicon mirror plate with a $5 \mathrm{~nm}$ thick layer of $\mathrm{Cr}$ is used as the reflector; the $\mathrm{Au}$ layer has a reflectivity of $95 \%$ at the $650 \mathrm{~nm}$ laser wavelength. In addition, the design of the OAWMM read/write head results in an optical spot $100 \mu \mathrm{m}$ in diameter projected onto the mirror plate. The mirror plate is oversized a bit to allow for reasonable alignment tolerances and thus the plate width is $170 \mu \mathrm{m}$ and its length in $220 \mu \mathrm{m}$.

Post-release flatness (radius of curvature greater than $10 \mathrm{~cm}$ ) of the mirror plate is achieved by a long high-temperature anneal $(5 \mathrm{hrs}$. at $1050 \mathrm{C})$. Stiffening ribs are fabricated integral to the mirror plate to prevent bimetallic distortion with temperature. Using the rib pattern as described in Figure 3 (perimeter of the mirror plate along with cross-bracing) wherein the ribs are $4 \mu \mathrm{m}$ wide and $6 \mu \mathrm{m}$ deep, center to edge distortion of the $\mathrm{Cr}-\mathrm{Au}$ mirror plate is only $28 \mathrm{~nm}$ for a temperature excursion of $50 \mathrm{C}$.

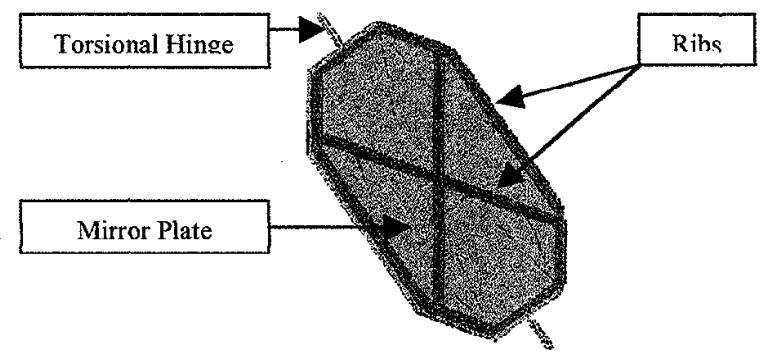

Figure 3. Solid model of backside of torsional mirror plate showing presence of stiffening ribs.

\section{Electro-Mechanical Properties}

The torsional mirror is driven using electrodes of polysilicon placed beneath the mirror plate. The two electrodes are on opposite sides of the rotational axis of the mirror plate. One of the electrodes is driven while the mirror plate and other electrode are grounded. The mirror plate rotates towards the driven electrode by a deflection angle $\theta$, which is established by the equilibrium between the electrostatic driving torque, $T_{e}$, and the spring restoring torque, $T_{s}$, provided by the torsional hinges. These torques are described in the following equations:

$$
\begin{aligned}
& T_{s}=k \theta \\
& T_{e}=\frac{V^{2}}{2} \frac{\partial C}{\partial \theta}
\end{aligned}
$$

where $k$ is the torsional spring constant supplied by the two hinges, $V$ is the voltage applied to the driven electrode, and $C$ is the capacitance between the grounded mirror plate and the driven electrode. For the simple case of a rectangular mirror plate having rectangular drive electrodes of length $a$ and width $b / 2$ in static deflection, the capacitance of the mirror is given by:

$$
C=\int_{0}^{\frac{b}{2}} \frac{\varepsilon_{0} a d x}{g-\theta x}=-\frac{\varepsilon_{0} a}{\theta} \ln \left(1-\frac{b \theta}{2 g}\right)
$$

where $\varepsilon_{0}$ is the permittivity of free space and $g$ is the air gap width of the undeflected mirror plate. Assuming $T_{e}=T_{s}$ and that there is no vertical translation of the mirror plate, Eqns. (1-3) can be solved analytically to determine the relationship between the actuation voltage and the mirror's deflection angle. Unfortunately this simple analytical approach does not correctly account for the nonrectangular shape of the mirror's plate and drive electrodes (see Fig. 3) nor for the presence of the stiffening ribs that renders the parallel plate capacitance analogy inappropriate. An alternative approach uses a finite element analysis (FEA) program (Pro/Mechanica ${ }^{\mathrm{TM}}$ ) to calculate the capacitance of the mirror plate as a function of angular rotation through the heat transfer analog for capacitance. For this situation it was found that the capacitance of the ribbed mirror could be empirically fit to the expression:

$$
C=-A \frac{\varepsilon_{0} a}{O} \ln \left(1-B \frac{b \theta}{2 g}\right)
$$

where $A$ and $B$ are fitting parameters than depend upon the precise geometry of the mirror plate. For the mirror plate of Figure 3, $A=0.85$ and $B=1.31$. With this revised expression for capacitance, the relationship between actuation voltage and angular deflection becomes:

$$
V=\sqrt{\left.\frac{2 k \theta^{3}}{\varepsilon_{0} c A A\left(\ln \left(1-B \frac{b \theta}{2 g}\right)+\frac{B \frac{b \theta}{2 g}}{1-B \frac{b \theta}{2 g}}\right.}\right)}
$$

Electrostatic pull-in occurs for the torsional mirror when the nonlinear electrostatic torque overwhelms the available restoring torque supplied by the hinges thus limiting the angular deflection range. This condition can be derived from the equilibrium state wherein the angular derivatives of the electrostatic and restoring torques are equal or, equivalently, the angular derivative of Eqn. (5) vanishes. The calculation leads to the pull-in condition:

$$
\begin{aligned}
& \alpha_{p i}=B \frac{b \theta_{p i}}{2 g}=0.44 \\
& V_{p t}=3.12 \sqrt{\frac{k}{\varepsilon_{0} a A} \alpha_{p t}\left(\frac{2 g}{B b}\right)^{3}}
\end{aligned}
$$

where $\theta_{p i}$ and $V_{p i}$ are the pull-in angle and voltage respectively. Eqns. (5-7) show that the presence of the stiffening ribs alters the available angular range and the necessary actuation voltage compared to the case of a flat rectangular mirror plate $(A=B=1)$.

The system response of the mirror to an applied electrostatic torque, $T_{e}$, can be described by a classical mass-spring-damper model:

$$
I \frac{d^{2} \theta}{d t^{2}}+t_{d} \frac{d \theta}{d t}+k \theta=T_{e}
$$

where $I$ is the mass moment of inertia of the mirror plate and $t_{d}$ represents the squeeze-film damping torque provided by the air gap. This equation can be rewritten as:

$$
\frac{d^{2} \theta}{d t^{2}}+\frac{\omega_{0}}{Q} \frac{d \theta}{d t}+\omega_{0}^{2} \theta=\frac{T_{e}}{I}
$$


where $Q$ represents the quality factor of this second order system and $\omega_{0}$ is the natural frequency of the system as defined by:

$$
\varpi_{0}=2 \pi f_{0}=\sqrt{\frac{k}{I}}
$$

Combining Eqn. (6), (7), and (10) provides an expression that describes the linear relationship between actuation voltage and bandwidth for a desired angular deflection range of the mirror:

$$
V_{p i}=19.6 f_{0} \sqrt{\frac{I}{\varepsilon_{0} a A} \theta_{p i}^{3}}=5.72 f_{0} \sqrt{\frac{I}{\varepsilon_{0} a A}\left(\frac{2 g}{B b}\right)^{3}}
$$

The mirror must be capable of steering the optical spot by \pm 4 data tracks (about $\pm 2.8 \mu \mathrm{m}$ ) corresponding to a mirror angular deflection of \pm 2 degrees. To provide some margin for error in the analytical model, we set the pull-in angle to be 2.6 degrees. The drive electrode dimensions are undersized from that of the mirror plate to allow for grounded landing pads beneath the perimeter stiffening ribs; thus $a=200 \mu \mathrm{m}$ and $b=140 \mu \mathrm{m}$. Applying Eqn. (6) then suggests that the initial air gap must be at least $9.5 \mu \mathrm{m}$; a target gap of $10 \mu \mathrm{m}$ was chosen for this device.

Servo system requirements dictate that the mirror have a resonance in excess of $25 \mathrm{kHz}$. Again using Pro/Mechanica ${ }^{\mathrm{TM}}$ FEA the resonant frequency can be calculated for the solid mode1 given a polysilicon modulus of $160 \mathrm{GPa}$ and a Poisson's ratio of 0.3. For the ribbed mirror plate geometry described previously and for torsional hinges $3.5 \mu \mathrm{m}$ wide by $25 \mu \mathrm{m}$ long and $2 \mu \mathrm{m}$ thick, FEA predicts a torsional resonance of $38 \mathrm{kHz}$ and a mass moment of inertia of $5 \times 10^{-13} \mathrm{~kg}-\mathrm{mm}^{3}$. Thus a pull-in voltage on the order of 142 Volts is expected.

\section{MURROR FABRICATION}

Fabrication of the micromachined mirror is described in the cross-sectional diagrams of Figure 4. To provide the high breakdown voltage required of the device, the silicon substrate is oxidized $(3000 \AA)$ and then a layer of LPCVD silicon nitride (2000 $\AA)$ is deposited on the oxide. ElectrodePoly $(2500 \AA)$ is then deposited and patterned to create interconnects, drive electrodes, and landing pads. A layer of PSG of a thickness equal to the ElectrodePoly layer thickness is then deposited and etched using a mask of opposite polarity to the ElectrodePoly mask. This reduces the topography of subsequent layers and consequently that of the mirror plate.

A thick $(9-10 \mu \mathrm{m})$ spacer layer of PSG is deposited then trenches are etched into it. Two trench etch steps are done, the first to rib depth $(6 \mu \mathrm{m})$ and then another to continue the trenches at the bond pads to the ElectrodePoly layer. All trenches are then filled with a $2.5 \mu \mathrm{m}$ thick conformal layer of TrenchPoly, which leaves severe cusps at their tops. To elminate these cusps a twostep chemical mechanical polish (CMP) is done using SDE3000 polysilicon slurry by Rodel. First the TrenchPoly is polished back to the top of the spacer PSG layer. Then another $2.5 \mu \mathrm{m}$ thick polysilicon layer (MirrorPoly) is deposited, annealed, and polished back to $2.0 \mu \mathrm{m}$ thick. The resulting surface of the MirrorPoly layer is smooth; RMS surface roughness is less than $3 \mathrm{~nm}$.

Next a $7000 \AA$ thick layer of $\mathrm{Au}$ with a $100 \AA$ thick $\mathrm{Cr}$ adhesion layer is deposited and patterned to provide wire bond pads. A second $\mathrm{Cr}-\mathrm{Au}$ layer is then deposited and patterned to create the reflector on the mirror plate. The MirrorPoly layer is then patterned to define the bond pads, mirror plate, and torsional hinges. Finally a HF release etch of the PSG spacer is done to free the mirror plate. A diced mirror chip ready for attaching to the $\mathrm{read} / \mathrm{write}$ head is shown in Figure 5.
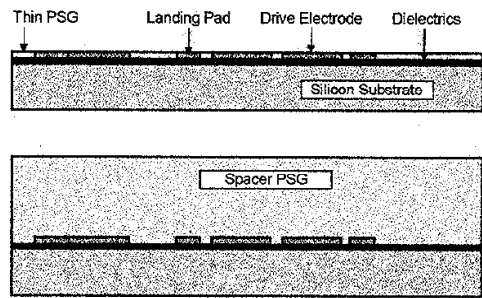

(B)
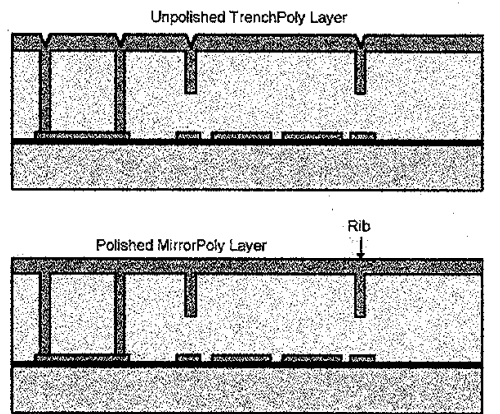

(D)

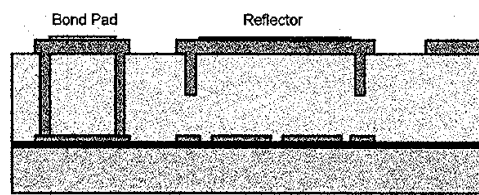

(E)

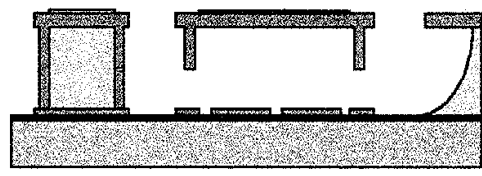

(F)

Figure 4. Fabrication sequence for mirror: (A) Thin PSG Pattern (B) Spacer PSG Deposition, (C) TrenchPoly Deposition, (D) Polished MirrorPoly, (E) Metallizations, and (F) Post-Release Etch.

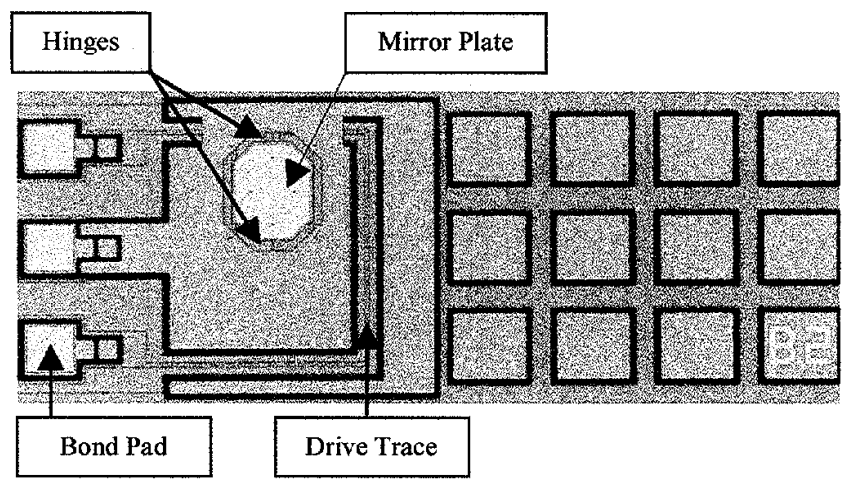

Figure 5. Silicon chip containing the micromachined torsional mirror. Square features at the right are stress relief mesas whereby the sacrificial PSG layer is removed between them.

\section{MIRROR TESTING}

The micromachined mirrors were tested using an optical approach similar to the one described in [8]. A collimated beam from a laser source is directed through a beam splitter and then 
focussed onto the reflecting surface of the mirror plate. The reflected beam is collimated upon returning through the focussing lens and is then redirected by 90 degrees by the beam splitter onto a Hamamatsu S2044 position sensitive detector (PSD). Upon angular rotation of the mirror plate, the reflected beam is displaced with respect to the incoming beam by an amount proportional to the focal length of the lens and the tangent of twice the angular deflection. The PSD's raw output signal is proportional to the laser spot's position on its active area. This output is conditioned using a Hamamatsu C4674 amplifier thus yielding a signal proportional to the angular displacement of the mirror plate. A fast digital oscilloscope (Tektronix 420A) is used to display and record the output of the amplifier.

Testing of the mirror involves procedures for measuring its angular displacement as a function of actuation voltage and for determining the mirror's frequency response. For both tests the mirror is driven single-sided with the undriven electrode and the mirror plate grounded. A high-voltage high-bandwidth amplifier whose input comes from a HP 33120A arbitrary waveform synthesizer supplies the mirror drive signal. For the angular deflection test, the drive signal to the mirror is a $500 \mathrm{~Hz}$ voltage ramping linearly from zero to 150 Volts. The amplified PSD output is then directly proportional to the deflection angle. For the frequency response test, the drive signal is a fast 100 Volt step. A fast Fourier transform (FFT) of the differentiated PSD output (step response) then yields the frequency response.

Figure 6 shows the performance of a typical torsional mirror showing an angular range of about 2 degrees before pull-in. Pullin voltage is about 145 volts. The mirror's frequency response is shown in Figure 7. Resonance occurs at about $36 \mathrm{kHz}$ with a $Q$ of about 5 .

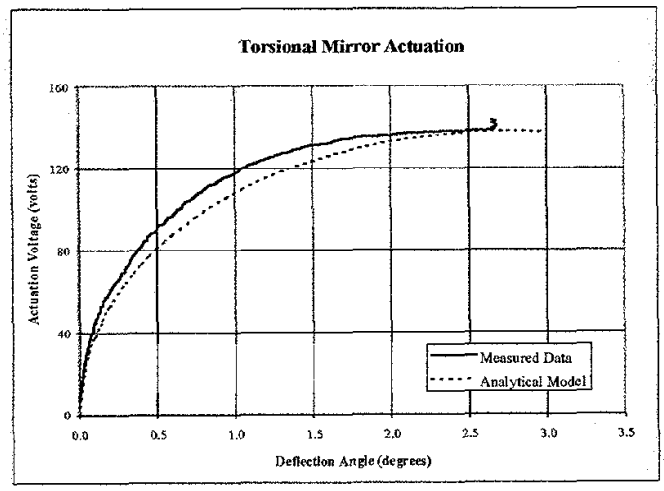

Figure 6. Actuation characteristics for a typical mirror compared to analytical model presented above.

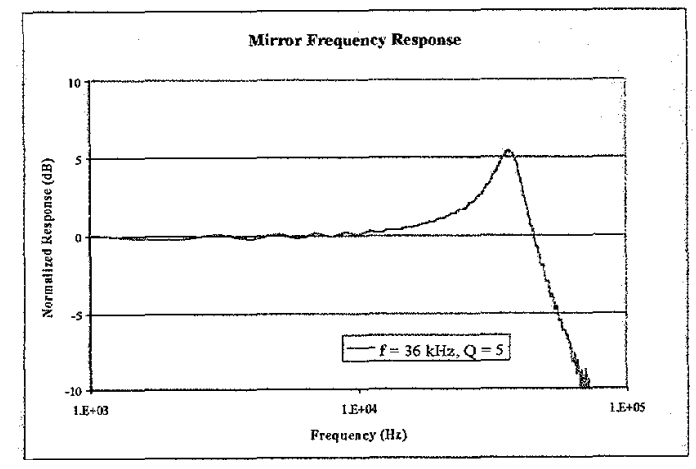

Figure 6. Torsional mirror frequency response.

\section{DISK DRIVE PERFORMANCE}

Operational read/write heads have been assembled and built into functioning prototypes of the OAW high bandwidth and relatively low $Q$ of the mirror allow for the successful implementation of the dual-stage servo system described above with a closed-loop bandwidth of $3-5 \mathrm{kHz}$. System simulations, however, suggest a maximum achievable servo bandwidth of $10 \mathrm{kHz}$. Although originally implemented for a data track density of $35 \mathrm{ktpi}$, the track misregistration error (TMR) for the system has been measured at $30 \mathrm{~nm}$ suggesting suitability for track densities as high as $100 \mathrm{ktpi}$.

Fine positioning by the mirror is also used to provide very fast short seeks. Prototype drives have demonstrated fully settled single-track seeks of $200 \mu \mathrm{sec}$. The performance for longer seeks are a function of the VCM coarse actuator; one-third stroke times are on the order of $13.5 \mathrm{msec}$. Given the small mass of the mirrors, the read/write head is quite robust to such accelerations.

\section{CONCLUSIONS}

Conventional surface micromachining techniques have been enhanced to create a polysilicon torsional mirror for second stage actuation in magneto-optical data storage. The design and fabrication process of the mirror have been optimized for optical efficiency and for electro-mechanical properties that allow for a high bandwidth servo loop within a Winchester-style disk drive. Prototypes of the disk drive have shown to be capable of supporting track densities on the order of $100 \mathrm{ktpi}$.

\section{ACKNOWLEDGEMENTS}

The authors would like to thank Kathy Jackson, Kevin Yasumura, Mark Walters, and Cronos Integrated Microsystems for help in fabrication and testing. Al Fennema was helpful in supplying data on the disk drive system-level performance.

\section{REFERENCES}

1. I. Naniwa, S. Nakamura, S. Saegusa, K. Sato, "Low Voltage Driven Piggy-Back Actuator of Hard Disk Drives", Proceedings of the $12^{\text {th }}$ Int. Conf. on MEMS (MEMS 99), Orlando, FL, pp. 49-52, 1999.

2. S. Kondo, S. Yoshimura, K. Yaguchi, Y. Miyata, J. Numazawa, M. Suzuki, M. Esashi, "Monolithic Piezo-Electric Actuator for Head Positioning", Transducers '99, Sendai, Japan, pp. 1838-1839, 1999.

3. T. Imamura, T. Koshikawa, M. Katayama, "Transverse Mode Electrostatic Microactuator for MEMS-based HDD Slider", Proceedings of the $9^{\text {th }}$ Int. Conf. on MEMS (MEMS 96), San Dicgo, CA, pp. 216-221, 1996.

4. D. Horsley, N. Wongkomet, R. Horowitz, A. Pisano, "Design and Feedback Control of Electrostatic Actuators for Magnetic Disk Drives", 1998 Solid-State Sensor and Actuator Workshop, Hilton Head, SC, pp. 120-123, 1998.

5. M. Sekimura, M. Yonezawa, K. Uchimaru, A. Kasahara, N. Uchida, "Electrostatic Torsion Mirror", Tech. Dig. of $16^{\text {th }}$ Sensor Symposium, pp. 167-170, 1998.

6. US Patent Nos. 5,850,375, 5,940,549, and 5,999,303.

7. J. Grade and H. Jerman, "A Large-Deflection Electrostatic Actuator for Optical Switching Applications", 2000 Solid State Sensor and Actuator Workshop, Hilton Head, SC, 2000.

8. K. Honer, N. Maluf, E. Martinez, G. Kovacs, "A HighResolution Laser-Based Deflection Measurement System for Characterizing Aluminum Electrostatic Actuators", Transducers' '95, Stockholm, Sweden, pp. 308-311, 1995. 